\title{
Stability Analysis and State Feedback Control of Networked Control Systems with Multi-Packet Transmission
}

\author{
Xun-Lin Zhu and Guang-Hong Yang
}

\begin{abstract}
This paper studies the problems of stability analysis and state feedback controller design for continuous-time networked control systems (NCSs). Model of NCSs with multiplepacket transmission and packet dropout in both the sensor-tocontroller channel and controller-to-actuator channel is derived, then the stability condition for NCSs with multiple-packet transmission is presented, and a new method for controller design is also proposed by using linear matrix inequality (LMI)based method. The simulation results illustrate the effectiveness of the proposed controller design for NCSs with multiple-packet transmission and packet dropout.
\end{abstract}

\section{INTRODUCTION}

As is well known, in modern industrial systems, sensors, controllers and plants are often connected over a network medium, such systems are usually called networked control systems (NCSs). There are many advantages in NCSs, such as low cost, reduced weight and power requirements, simple installation and maintenance, and high reliability. Thus, increasing research interests have been paid to the study of the stability and stabilization of NCSs.

By decomposing network-induced delays into fixed and varying parts, the NCSs were modeled as parameteruncertain systems and controller design methods based on delay-dependent stability conditions were presented (see [1][2]). However, the time delay considered in [1] was shorter than a sampling period. [3] designed the stochastic optimal controllers for networked control systems with networkinduced delay longer than a sampling period. By using Lyapunov-Razumikhin function and Lyapunov-Krasovskii function methods, [4] was dedicated to the design of state feedback controllers and the admissible upper bounds of time delay and packet dropout were also presented. In [5], a model of the NCSs was provided under consideration of both the

This work was supported in part by Program for New Century Excellen Talents in University (NCET-04-0283), the Funds for Creative Research Groups of China (No. 60521003), Program for Changjiang Scholars and Innovative Research Team in University (No. IRT0421), the State Key Program of National Natural Science of China (Grant No. 60534010), the Funds of National Science of China (Grant No. 60674021) and the Funds of PhD program of MOE, China (Grant No. 20060145019), the 111 Project (B08015).

Xun-Lin Zhu is with the College of Information Science and Engineering, Northeastern University, Shenyang, Liaoning, 110004, China. He is also with the School of Computer and Communication Engineering, Zhengzhou University of Light Industry, Zhengzhou, Henan, 450002, China. hnt jxx@163.com

Guang-Hong Yang is with the College of Information Science and Engineering, Northeastern University, Shenyang, Liaoning, 110004, China. He is also with the Key Laboratory of Integrated Automation of Process Industry, Ministry of Education, Northeastern University, Shenyang 110004, China. Corresponding author. yangguanghonglise.neu.edu.cn, yang_guanghong@163. com network-induced delay and the data packet dropout. For other results dealing with time delay and packet dropout, see also [6]-[9]. The problem of $H_{\infty}$ controller design for NCSs was considered in [10].

All works mentioned above discussed the case of singlepacket transmission of NCSs. However, especially in distributed NCSs, once the length of sampled data surpasses the maximum admissible length of the network packet, multiplepacket transmission is ubiquitous (such as the systems based on Device-Net). For this case, the sampled data and control inputs are split into multiple separated packets which may not arrive at the destination simultaneously. Obviously, the NCSs with single-packet transmission is a subclass of the NCSs with multiple-packet transmission.

As for the problem of multiple-packet transmission, to the best of our knowledge, few papers discussed it thoroughly except in [11] and [12]. As one can see, [11] presented the discretized model of MIMO NCS with multiple time delays, and the closed-loop NCS model only included a standard controller designed without considering the time delay effect a priori. [12] considered the case that multiplepacket transmission exists only in sensor-to-controller channel and the controller-to-actuator channel must be singlepacket transmission, if multiple-packet transmission also exists in controller-to-actuator channel, the result of [12] is not applicable.

This paper presents a model of NCSs with multiplepacket transmission and packet dropout in both the sensorto-controller channel and the controller-to-actuator channel, the stability analysis and state feedback controller design are also presented by using LMI-based method (see [13]). Three examples are finally given to illustrate the effectiveness and less conservatism of our method.

\section{MODELING OF NCSS AND PRELIMINARIES}

Throughout this paper, we assume that the sensor is clockdriven, the controller and actuator are event-driven (realized via zero-order holders). The controller and the actuator will be updated until the new data packet arrives. Denote $T$ as the length of sampling period.

Under a linear control law, the controlled system can be expressed as

$$
\begin{aligned}
& \dot{x}(t)=A x(t)+B u(t), \\
& x(t)=\phi(t), t<0, \\
& u^{C}(t)=K x^{C}(t),
\end{aligned}
$$

where $x(t) \in \mathfrak{R}^{n}, u(t) \in \mathfrak{R}^{p}, A$ and $B$ are constant matrices of appropriate dimensions. $\phi(t)$ is a continuously differentiable initial function. $x^{C}$ is the delayed version of $x, u$ is the 
delayed version of $u^{C}$, and $x^{C}$ and $u^{C}$ are the input and the output of controller, respectively.

The plant state is split into $r$ parts

$$
x(t)=\left[\begin{array}{lll}
X_{1}^{T}(t) & \cdots & X_{r}^{T}(t)
\end{array}\right]^{T},
$$

and every part with its time stamp is lumped into a packet. Similarly, the control signal is split into $s$ parts

$$
u^{C}(t)=\left[\begin{array}{lll}
U_{1}^{C}(t)^{T} & \cdots & U_{s}^{C}(t)^{T}
\end{array}\right]^{T},
$$

and every part with its time stamp is also lumped into a packet, where $X_{i}(t) \in \Re^{r_{i}}, r_{i} \in Z^{+}$, and $\sum_{i=1}^{r} r_{i}=n ; U_{i}^{C}(t) \in$ $\mathfrak{R}^{s_{i}}, s_{i} \in Z^{+}$, and $\sum_{i=1}^{s} s_{i}=p$.

For simplicity, we consider the case that the plant state and the control signal are split into two parts, respectively. Fig. 1 illustrates the model of NCSs with multiple packets transmission.

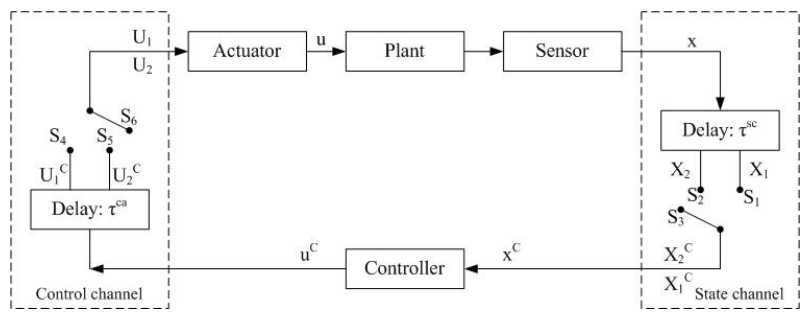

Fig. 1. An NCS with multiple-packet transmission

Just as shown in Fig. 1, at the instant $t$, the state data packets, which are waiting for transferring, in $S_{1}, S_{2}$ are $X_{1}\left(k_{i_{1}} T\right)$ and $X_{2}\left(k_{i_{2}} T\right)\left(k_{i_{1}}, k_{i_{2}} \in Z^{+}\right)$, respectively. Meanwhile, the controller input data packets $X_{1}^{C}(t), X_{2}^{C}(t)$, which are waiting to be updated, are equal to $X_{1}\left(k_{1} T\right), X_{2}\left(k_{2} T\right)\left(k_{1}, k_{2} \in\right.$ $\left.Z^{+}\right)$, respectively. Throughout this paper, we suppose the controller will calculate the control inputs based on the latest available plant states. Thus, the process of state data transmission can be described as follows:

Case 1: If the switch is in position $S_{3}$, i.e., the state data packets $X_{1}\left(k_{i_{1}} T\right)$ and $X_{2}\left(k_{i_{2}} T\right)$ are dropped in the sensor-tocontroller channel, then

$$
X_{1}^{C}(t)=X_{1}\left(k_{1} T\right), \quad X_{2}^{C}(t)=X_{2}\left(k_{2} T\right) .
$$

Case 2: If the switch is in position $S_{2}$, then

$$
X_{1}^{C}(t)=X_{1}\left(k_{1} T\right), \quad X_{2}^{C}(t)=\left\{\begin{array}{cl}
X_{2}\left(k_{i_{2}} T\right), & \text { if } k_{i_{2}}>k_{2}, \\
X_{2}\left(k_{2} T\right), & \text { otherwise. }
\end{array}\right.
$$

Case 3 : If the switch is in position $S_{1}$, then

$$
X_{2}^{C}(t)=X_{2}\left(k_{2} T\right), \quad X_{1}^{C}(t)=\left\{\begin{array}{cc}
X_{1}\left(k_{i_{1}} T\right), & \text { if } k_{i_{1}}>k_{1}, \\
X_{1}\left(k_{1} T\right), & \text { otherwise. }
\end{array}\right.
$$

Therefore, there exist $k_{j_{1}}, k_{j_{2}} \in Z^{+}$, such that

$$
x^{C}(t)=\left[\begin{array}{c}
X_{1}^{C}(t) \\
X_{2}^{C}(t)
\end{array}\right]=\left[\begin{array}{c}
X_{1}\left(k_{j_{1}} T\right) \\
X_{2}\left(k_{j_{2}} T\right)
\end{array}\right] .
$$

Define $\tau^{s c}(t)=\left[\begin{array}{c}\tau_{1}^{s c}(t) \\ \tau_{2}^{s c}(t)\end{array}\right]$, where $\tau_{i}^{s c}(t)=t-k_{j_{i}} T(i=1,2)$, then $\tau_{i}^{s c}$ represents the delay of the $i$ th state data part, and

$$
x^{C}(t)=\left[\begin{array}{c}
X_{1}\left(t-\tau_{1}^{s c}(t)\right) \\
X_{2}\left(t-\tau_{2}^{s c}(t)\right)
\end{array}\right] .
$$

The process of control signal transmission is similar to the process of state data transmission, here it is omitted.

Denote $t_{k}\left(k \in Z^{+}\right)$as the instant that the actuator receives the $k$ th control signal, $t_{k}^{C}\left(k \in Z^{+}\right)$as the instant that the controller sends the $k$ th control signal to the actuator. Suppose $u\left(t_{k}^{+}\right)$is based on the outputs of controller at the instant $t_{k_{1}}^{C}$ and $t_{k_{2}}^{C}$, respectively, i.e.,

$$
\begin{aligned}
u\left(t_{k}^{+}\right) & =\left[\begin{array}{l}
U_{1}\left(t_{k}^{+}\right) \\
U_{2}\left(t_{k}^{+}\right)
\end{array}\right]=\left[\begin{array}{c}
U_{1}^{C}\left(t_{k^{C}}^{C}\right) \\
U_{2}^{C}\left(t_{k_{2}}^{C}\right)
\end{array}\right] \\
& =D_{1} u^{C}\left(t_{k_{1}}^{C}\right)+D_{2} u^{C}\left(t_{k_{2}}^{C}\right),
\end{aligned}
$$

where

$$
D_{1}=\operatorname{diag}\left\{I_{s_{1}}, 0\right\}, D_{2}=\operatorname{diag}\left\{0, I_{s_{2}}\right\} .
$$

Similarly, the input of controller at the instant $t_{k_{1}}^{C}$ is based on the states of plant at the instant $k_{1}^{1} T$ and $k_{1}^{2} T$, the input of controller at the instant $t_{k_{2}}^{C}$ is based on the states of plant at the instant $k_{2}^{1} T$ and $k_{2}^{2} T$, respectively, i.e.,

$$
\begin{aligned}
x^{C}\left(t_{k_{1}}^{C}\right) & =\left[\begin{array}{l}
X_{1}^{C}\left(t_{k_{1}}^{C}\right) \\
X_{2}^{C}\left(t_{k_{1}}^{C}\right)
\end{array}\right]=\left[\begin{array}{l}
X_{1}\left(k_{1}^{1} T\right) \\
X_{2}\left(k_{1}^{2} T\right)
\end{array}\right] \\
& =C_{1} x\left(k_{1}^{1} T\right)+C_{2} x\left(k_{1}^{2} T\right), \\
x^{C}\left(t_{k_{2}}^{C}\right) & =\left[\begin{array}{l}
X_{1}^{C}\left(t_{k_{2}}^{C}\right) \\
X_{2}^{C}\left(t_{k_{2}}^{C}\right)
\end{array}\right]=\left[\begin{array}{l}
X_{1}\left(k_{2}^{1} T\right) \\
X_{2}\left(k_{2}^{2} T\right)
\end{array}\right] \\
& =C_{1} x\left(k_{2}^{1} T\right)+C_{2} x\left(k_{2}^{2} T\right),
\end{aligned}
$$

where

$$
C_{1}=\operatorname{diag}\left\{I_{r_{1}}, 0\right\}, C_{2}=\operatorname{diag}\left\{0, I_{r_{2}}\right\},
$$

and $k_{1}^{1}, k_{1}^{2}, k_{2}^{1}, k_{2}^{2} \in Z^{+}$.

Thus, for $t \in\left[t_{k}, t_{k+1}\right)$, we obtain

$$
\begin{aligned}
u(t)=u\left(t_{k}^{+}\right)= & D_{1} K C_{1} x\left(k_{1}^{1} T\right)+D_{1} K C_{2} x\left(k_{1}^{2} T\right) \\
& +D_{2} K C_{1} x\left(k_{2}^{1} T\right)+D_{2} K C_{2} x\left(k_{2}^{2} T\right) .
\end{aligned}
$$

Denote $t-k_{j}^{i} T$ as $\tau_{i j}(t)$, i.e,

$$
\tau_{i j}(t)=t-k_{j}^{i} T, \quad \forall t \in\left[t_{k}, t_{k+1}\right),
$$

analogous to the case of two-packet transmission, for the case of multiple-packet transmission, for $t \in\left[t_{k}, t_{k+1}\right)$, we have

$$
\begin{aligned}
& u(t)=\sum_{j=1}^{s} \sum_{i=1}^{r} D_{j} K C_{i} x\left(k_{j}^{i} T\right)=\sum_{j=1}^{s} \sum_{i=1}^{r} D_{j} K C_{i} x\left(t-\tau_{i j}(t)\right), \\
& C_{i}=\operatorname{diag}\{\underbrace{0, \cdots, I_{r_{i}}}_{i}, \cdots, 0\} \text {, } \\
& D_{j}=\operatorname{diag}\{\underbrace{0, \cdots, I_{s_{j}}}_{j}, \cdots, 0\} .
\end{aligned}
$$


Assumption 1. The pair $(A, B)$ is stabilizable.

Assumption 2. There exist constants $h_{i j}$ such that

$$
0 \leq \tau_{i j}(t) \leq h_{i j}, \quad \forall i=1,2, \cdots, r, j=1,2, \cdots, s .
$$

Denoting $h=\max _{1 \leq i \leq r, 1 \leq j \leq s}\left\{h_{i j}\right\}$, then the model of NCSs with multiple-packet transmission under Assumption 1 and 2 can be described as

$$
\begin{aligned}
& \dot{x}(t)=A x(t)+\sum_{j=1}^{s} \sum_{i=1}^{r} B D_{j} K C_{i} x\left(t-\tau_{i j}(t)\right) \\
& x(t)=\phi(t), \quad t \in[-h, 0] \\
& 0 \leq \tau_{i j}(t) \leq h_{i j}, \quad i=1,2, \cdots, r ; j=1,2, \cdots, s .
\end{aligned}
$$

For convenience of analysis, the system (12)-(14) can be rewritten as follows:

$$
\begin{aligned}
& \dot{x}(t)=A x(t)+\sum_{l=1}^{m} A_{l} x\left(t-\tau_{l}(t)\right), \\
& x(t)=\phi(t), \quad t \in[-h, 0] \\
& 0 \leq \tau_{l}(t) \leq h_{l}, \quad l=1,2, \cdots, m .
\end{aligned}
$$

where

$$
\begin{aligned}
& A_{l}=B D_{j} K C_{i}, \tau_{l}(t)=\tau_{i j}(t), h_{l}=h_{i j}, \\
& \forall l=(j-1) \times r+i, i=1,2, \cdots, r ; j=1,2, \cdots, s .
\end{aligned}
$$

In this paper, we will analyze the stability criteria of the closed-loop system (15)-(17) and study the problem of state-feedback controller design.

Remark 1. If $m=1$, then the system (15)-(17) is the same as the system with single-packet transmission which has been considered in [5], for convenience of comparison, the main result (Corollary 1) in [5] is listed as follows.

Lemma 1. [5] The system (15)-(17) with $m=1$ is asymptotically stable if there exist matrices $P>0, T>0$, and matrices $X_{i}(i=1,2,3)$ such that

$$
\Lambda=\left[\begin{array}{cccc}
\Lambda_{11} & \Lambda_{12} & N_{3}^{T}-\left(X_{3} A\right)^{T}+X_{1}+P & h_{1} N_{1} \\
* & \Lambda_{22} & -N_{3}^{T}+X_{2}-\left(X_{3} B K\right)^{T} & h_{1} N_{2} \\
* & * & X_{3}+X_{3}^{T}+h_{1} T & h_{1} N_{3} \\
* & * & * & -h_{1} T
\end{array}\right]<0
$$

where

$$
\begin{aligned}
& \Lambda_{11}=N_{1}+N_{1}^{T}-X_{1} A-\left(X_{1} A\right)^{T}, \\
& \Lambda_{12}=N_{2}^{T}-N_{1}-\left(X_{2} A\right)^{T}-M_{1} B K, \\
& \Lambda_{22}=-N_{2}-N_{2}^{T}-X_{2} B K-\left(X_{2} B K\right)^{T} .
\end{aligned}
$$

\section{MAIN RESULTS}

In this section, we analyze asymptotic stability of the closed-loop system (15)-(17), and propose a method of designing state feedback controllers.

\section{A. Stability Analysis}

Theorem 1. The system (15)-(17) is asymptotically stable if there exist symmetrical matrices $P>0, R_{i} \geq 0, S_{i}>0$ and matrices $M_{1}, M_{2}, \cdots, M_{2 m+2}$, such that

$$
\Omega<0,
$$

where

$$
\begin{aligned}
& \Omega=\left[\begin{array}{cc}
\Omega_{1} & \Omega_{2} \\
* & \Omega_{3}
\end{array}\right]-M \mathscr{A}-(M \mathscr{A})^{T}, \\
& \begin{array}{l}
\Omega_{1}=\left[\begin{array}{cc}
\sum_{i=1}^{m}\left(R_{i}-S_{i}\right) & P \\
* & \sum_{i=1}^{m} h_{i}^{2} S_{i}
\end{array}\right], \\
\Omega_{2}=\left[\begin{array}{llll}
\Omega_{21} & \Omega_{22} & \cdots & \Omega_{2 m}
\end{array}\right],
\end{array} \\
& \Omega_{2 i}=\left[\begin{array}{cc}
S_{i} & 0 \\
0 & 0
\end{array}\right](i=1,2, \cdots, m) \text {, } \\
& \Omega_{3}=\operatorname{diag}\left\{\Omega_{31}, \Omega_{32}, \cdots, \Omega_{3 m}\right\} \text {, } \\
& \Omega_{3 i}=\left[\begin{array}{cc}
-2 S_{i} & S_{i} \\
* & -R_{i}-S_{i}
\end{array}\right](i=1,2, \cdots, m) \text {, } \\
& M=\left[\begin{array}{llll}
M_{1}^{T} & M_{2}^{T} & \cdots & M_{2 m+2}^{T}
\end{array}\right]^{T}, \\
& \mathscr{A}=\left[\begin{array}{lllllll}
A & -I & A_{1} & 0 & \cdots & A_{m} & 0
\end{array}\right] .
\end{aligned}
$$

Proof: Construct a Lyapunov functional as

$$
V(t)=V_{1}(t)+V_{2}(t)+V_{3}(t)
$$

with

$$
\begin{aligned}
& V_{1}(t)=x^{T}(t) \operatorname{Px}(t), \\
& V_{2}(t)=\sum_{i=1}^{m} \int_{t-h_{i}}^{t} x^{T}(s) R_{i} x(s) d s, \\
& V_{3}(t)=\sum_{i=1}^{m} \int_{-h_{i}}^{0} \int_{t+\beta}^{t} \dot{x}^{T}(s) h_{i} S_{i} \dot{x}(s) d s d \beta .
\end{aligned}
$$

Using the Cauchy-Schwarz inequality [14], and denoting $\zeta=\left[x^{T}(t), \dot{x}^{T}(t), x^{T}\left(t-\tau_{1}(t)\right), x^{T}\left(t-h_{1}\right), \cdots, x^{T}(t-\right.$ $\left.\left.\tau_{m}(t)\right), x^{T}\left(t-h_{m}\right)\right]^{T}$, one gets

$$
\dot{V}(t) \leq \zeta^{T} \Omega \zeta
$$

So, it is not difficult to see that (20) guarantees $\dot{V}(t)<0$, this immediately implies the asymptotic stability of the system (15)-(17), thus it completes the proof.

Remark 2. By using the Cauchy-Schwarz inequality, a stability condition of the system (15)-(17) is derived in Theorem 1. Since the Newton-Leibniz formula is not employed, the structure of the derived inequality in Theorem 1 is simple. In addition, there are some redundant variables among $M_{i}(i=1,2, \cdots, 2 m+2)$, which will be verified in the following.

By using the elimination Lemma ([13], p.22), $\Omega<0$ is equivalent to

$$
\Phi=\mathscr{N}_{\mathscr{A}}^{T} \Omega \mathscr{N}_{\mathscr{A}}<0,
$$

where $\mathscr{N}_{\mathscr{A}}$ denotes the full-rank matrix representations of the right annihilator of $A$.

By the Schur complement, $\Phi<0$ is equivalent to

$$
\bar{\Phi}<0
$$


where

$$
\begin{aligned}
& \bar{\Phi}=\left[\begin{array}{cc}
\Omega_{1} & \Omega_{2} \\
* & \Omega_{3}
\end{array}\right]-\tilde{M} \mathscr{A}-(\tilde{M} \mathscr{A})^{T}, \\
& \tilde{M}=\left[\begin{array}{ccccc}
P_{1} & -\sum_{i=1}^{m} h_{i}^{2} S_{i} & 0 & \ldots & 0
\end{array}\right]^{T},
\end{aligned}
$$

and $\Omega_{1}, \Omega_{2}, \Omega_{3}, \mathscr{A}$ are defined in (20). So, it follows that $\Omega<0$ in (20) is equivalent to $\bar{\Phi}<0$ in (25).

Comparing with (25), it is easy to see that $M_{3}, M_{4}, \cdots, M_{2 m+2}$ are all redundant in (20). So, the simplified form of Theorem 1 is given as follows.

Theorem 2. The system (15)-(17) is asymptotically stable if there exist symmetrical matrices $P>0, R_{i} \geq 0, S_{i}>0$ and matrices $M_{1}, M_{2}$, such that

$$
\tilde{\Omega}<0,
$$

where

$$
\begin{aligned}
& \tilde{\Omega}=\left[\begin{array}{cc}
\Omega_{1} & \Omega_{2} \\
* & \Omega_{3}
\end{array}\right]-M \mathscr{A}-(M \mathscr{A})^{T}, \\
& M=\left[\begin{array}{ccccc}
M_{1}^{T} & M_{2}^{T} & 0 & \ldots & 0
\end{array}\right]^{T},
\end{aligned}
$$

and $\Omega_{1}, \Omega_{2}, \Omega_{3}, \mathscr{A}$ are defined in (20).

From Theorem 2, we can derive a stability condition for the system (15)-(17) with $m=1$ as follows.

Corollary 1. For given scalar $h_{1}$, the system (15)-(17) with $m=1$ is asymptotically stable if there exist symmetric matrices $P>0, R \geq 0, S>0$ and matrices $M_{1}, M_{2}$, such that

$$
\Theta=\left[\begin{array}{cc}
\Theta_{1} & \Theta_{2} \\
* & \Theta_{3}
\end{array}\right]<0
$$

where

$$
\begin{aligned}
& \Theta_{1}=\left[\begin{array}{cc}
R-S-M_{1} A-\left(M_{1} A\right)^{T} & P+M_{1}-\left(M_{2} A\right)^{T} \\
* & h_{1}^{2} S+M_{2}+M_{2}^{T}
\end{array}\right], \\
& \Theta_{2}=\left[\begin{array}{cc}
S-M_{1} B K & 0 \\
-M_{2} B K & 0
\end{array}\right], \\
& \Theta_{3}=\left[\begin{array}{cc}
-2 S & S \\
* & -R-S
\end{array}\right] \text {. }
\end{aligned}
$$

Now, by comparing Corollary 1 and Lemma 1, we can get the following result.

Theorem 3. If $\Lambda<0$ in Lemma 1 is feasible, $\Theta<0$ in Corollary 1 is also feasible.

Proof: Pre- and post-multiplying both sides of $\Lambda$ with

$$
\Pi_{1}=\left[\begin{array}{cccc}
I & 0 & 0 & -h_{1}^{-1} I \\
0 & I & 0 & h_{1}^{-1} I \\
0 & 0 & I & 0 \\
0 & 0 & 0 & I
\end{array}\right]
$$

and its transpose, it follows that

$$
\Lambda_{1}<0
$$

where

$$
\begin{aligned}
\Lambda_{1}= & {\left[\begin{array}{ccc}
-h_{1}^{-1} T & h_{1}^{-1} T & P \\
* & -h_{1}^{-1} T & 0 \\
* & * & h_{1} T
\end{array}\right]-X\left[\begin{array}{lll}
A & B K & -I
\end{array}\right] } \\
& \quad-\left(X\left[\begin{array}{lll}
A & B K & -I
\end{array}\right]\right)^{T}, \\
X= & {\left[\begin{array}{lll}
X_{1}^{T} & X_{2}^{T} & X_{3}^{T}
\end{array}\right]^{T} . }
\end{aligned}
$$

By using the elimination Lemma and the Schur complement, $\Lambda_{1}<0$ is equivalent to

$$
\Lambda_{2}<0
$$

where

$$
\Lambda_{2}=\left[\begin{array}{ccc}
-h_{1}^{-1} T+P A+A^{T} P & h_{1}^{-1} T+P B K & h_{1} A^{T} T \\
* & -h_{1}^{-1} T & h_{1}(B K)^{T} T \\
* & * & -h_{1} T
\end{array}\right] .
$$

Let

$$
R=0, S=h_{1}^{-1} T, M_{1}=-P, M_{2}=-h_{1} T,
$$

then $\Theta$ in Corollary 1 becomes

$$
\Lambda_{3}=\left[\begin{array}{cccc}
\Lambda_{31} & h_{1} A^{T} T & h_{1}^{-1} T+P B K & 0 \\
* & -h_{1} T & h_{1} T B K & 0 \\
* & * & -2 h_{1}^{-1} T & h_{1}^{-1} T \\
* & * & * & -h_{1}^{-1} T
\end{array}\right]
$$

where

$$
\Lambda_{31}=-h_{1}^{-1} T+P A+A^{T} P .
$$

Note that

$$
\Pi_{2} \Lambda_{3} \Pi_{2}^{T}=\left[\begin{array}{cc}
\Lambda_{2} & 0 \\
* & -h_{1}^{-1} T
\end{array}\right]<0
$$

where

$$
\Pi_{2}=\left[\begin{array}{llll}
I & 0 & 0 & 0 \\
0 & 0 & I & I \\
0 & I & 0 & 0 \\
0 & 0 & 0 & I
\end{array}\right]
$$

so, $\Lambda_{2}<0$ is equivalent to $\Lambda_{3}<0$. This implies that $\Theta<0$ in Corollary 1 is feasible.

Remark 3. Theorem 3 shows that Theorem 2 with $m=1$ is less conservative than Lemma 1.

\section{B. Stabilization of NCSs via State Feedback}

Based on Theorem 2, we are now in a position to design the feedback gain $K$ to render system (15)-(17) asymptotically stable.

Obviously, (26) implies $M_{2}$ is nonsingular, so there exists a matrix $U$, such that $M_{1}=M_{2} U$. Denoting

$$
\begin{aligned}
& \bar{M}_{2}=M_{2}^{-1}, \bar{P}=\bar{M}_{2} P \bar{M}_{2}^{T}, \bar{R}_{i}=\bar{M}_{2} R_{i} \bar{M}_{2}^{T}, \\
& \bar{S}_{i}=\bar{M}_{2} S_{i} \bar{M}_{2}^{T}, \quad \forall i=1,2, \cdots, m,
\end{aligned}
$$

we can obtain

$$
A_{l} \bar{M}_{2}^{T}=B D_{j} K C_{i} \bar{M}_{2}^{T}
$$

where $l=(j-1) \times r+i, j=1,2, \cdots, s, i=1,2, \cdots, r$. If

$$
\bar{M}_{2}=\operatorname{diag}\left\{M_{21}, M_{22}, \cdots, M_{2 r}\right\}
$$


where $M_{2 i} \in \mathfrak{R}^{r_{i} \times r_{i}}(i=1,2, \cdots, r)$, then

$$
\begin{aligned}
C_{i} \bar{M}_{2}^{T}= & \operatorname{diag}\{\underbrace{0, \cdots, I_{r_{i}}}_{i}, \cdots, 0\} \\
& \times \operatorname{diag}\left\{M_{21}^{T}, M_{22}^{T}, \cdots, M_{2 r}^{T}\right\} \\
= & \operatorname{diag}\{\underbrace{0, \cdots, M_{2 i}^{T}}_{i}, \cdots, 0\} .
\end{aligned}
$$

Partitioning $K$ as

$$
K=\left[\begin{array}{llll}
K_{1} & K_{2} & \cdots & K_{r}
\end{array}\right]
$$

and denoting

$$
G_{i}=\left[\begin{array}{lllll}
\underbrace{0}_{i} & \cdots & I_{r_{i}} & \cdots & 0
\end{array}\right],
$$

where $K_{i} \in \mathfrak{R}^{p \times r_{i}}(i=1,2, \cdots, r)$, and introducing new variables $F_{i}=K_{i} M_{2 i}^{T}(i=1,2, \cdots, r)$, it is easy to see that

$$
\begin{aligned}
A_{l} \bar{M}_{2}^{T} & =B D_{j} K C_{i} \bar{M}_{2}^{T} \\
& =B D_{j} \times\left[\begin{array}{llll}
\underbrace{0}_{i} \cdots & F_{i} & \cdots & 0
\end{array}\right] \\
& =B D_{j} F_{i} G_{i},
\end{aligned}
$$

and

$$
\begin{aligned}
\bar{M}_{2}^{T} & =\operatorname{diag}\left\{M_{21}^{T}, M_{22}^{T}, \cdots, M_{2 r}^{T}\right\} \\
& =\sum_{i=1}^{r} \operatorname{diag}\{\underbrace{0, \cdots, M_{2 i}^{T}}_{i}, \cdots, 0\} \\
& =\sum_{i=1}^{r} G_{i}^{T} M_{2 i}^{T} G_{i},
\end{aligned}
$$

pre- and post-multiplying both sides of $\tilde{\Omega}$ in (26) with $\operatorname{diag}\left\{\bar{M}_{2}, \bar{M}_{2}, \cdots, \bar{M}_{2}\right\}$ and its transpose, we can obtain the following theorem.

Theorem 4. For given $h_{i}(i=1,2, \cdots, m)$ and $U$, the system (15)-(17) is asymptotically stable if there exist symmetrical matrices $\bar{P}>0, \bar{R}_{i} \geq 0, \bar{S}_{i}>0(i=1,2, \cdots, m)$ and matrices $F_{j}, M_{2 j}(j=1,2, \cdots, r)$, such that

$$
\bar{\Omega}<0,
$$

where

$$
\begin{aligned}
& \bar{\Omega}=\left[\begin{array}{cc}
\bar{\Omega}_{1} & \bar{\Omega}_{2} \\
* & \bar{\Omega}_{3}
\end{array}\right]-\bar{M} \overline{\mathscr{A}}-(\bar{M} \overline{\mathscr{A}})^{T},
\end{aligned}
$$

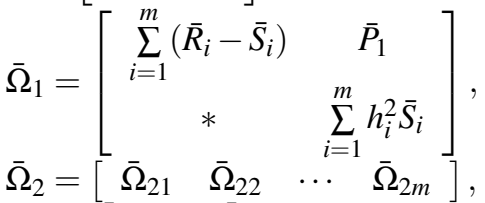

$$
\begin{aligned}
& \bar{\Omega}_{2 i}=\left[\begin{array}{cc}
\bar{S}_{i} & 0 \\
0 & 0
\end{array}\right](i=1,2, \cdots, m) \text {, } \\
& \bar{\Omega}_{3}=\operatorname{diag}\left\{\bar{\Omega}_{31}, \bar{\Omega}_{32}, \cdots, \bar{\Omega}_{3 m}\right\},
\end{aligned}
$$

$$
\begin{aligned}
& \bar{\Omega}_{3 i}=\left[\begin{array}{cc}
-2 \bar{S}_{i} & \bar{S}_{i} \\
* & -\bar{R}_{i}-\bar{S}_{i}
\end{array}\right](i=1,2, \cdots, m), \\
& \bar{M}=\left[\begin{array}{lllll}
U^{T} & I & 0 & \cdots & 0
\end{array}\right]^{T}, \\
& \overline{\mathscr{A}}=\left[\begin{array}{lllllll}
H_{1} & H_{2} & N_{1} & 0 & \cdots & N_{m} & 0
\end{array}\right], \\
& H_{1}=A \sum_{i=1}^{r} G_{i}^{T} M_{2 i}^{T} G_{i}, \\
& H_{2}=-\sum_{i=1}^{i} G_{i}^{T} M_{2 i}^{T} G_{i}, \\
& N_{l}=B D_{j} F_{i} G_{i}, \\
& \forall l=(j-1) \times r+i, i=1,2, \cdots, r ; j=1,2, \cdots, s .
\end{aligned}
$$

The state-feedback gain is then given by

$$
\begin{aligned}
K= & {\left[\begin{array}{llll}
F_{1} & F_{2} & \cdots & F_{r}
\end{array}\right] } \\
& \times \operatorname{diag}\left\{M_{21}^{-T}, M_{22}^{-T}, \cdots, M_{2 r}^{-T}\right\} .
\end{aligned}
$$

Remark 4. In contrast with the controller design method given in [12], Theorem 4 involves slack variables $M_{1}, M_{2}$. By defining $M_{1}=M_{2} U$, the controller gain $K$ can be obtained by solving a set of LMIs. However, the stabilization result in [12] is only applicable to the case of multiple-packet transmission occurred in state channel and single-packet transmission in control channel. In addition, the restrict $P B=B M$ in [12] is not needed in Theorem 4, which will introduce less conservatism. An example in the following section will verify this fact.

Corollary 2. For given scalar $h_{1}$ and matrices $U$, the system (15)-(17) with $m=1$ is asymptotically stable if there exist symmetric matrices $\bar{P}>0, \bar{R} \geq 0, \bar{S}>0$ and matrices $F, M_{2}$, such that

$$
\Psi=\left[\begin{array}{cc}
\Psi_{1} & \Psi_{2} \\
* & \Psi_{3}
\end{array}\right]<0,
$$

where

$$
\begin{aligned}
& \Psi_{1}=\left[\begin{array}{cc}
\bar{R}-\bar{S}-U A M_{2}^{T}-M_{2} A^{T} U^{T} & \bar{P}+U M_{2}^{T}-M_{2} A^{T} \\
* & h_{1}^{2} S+M_{2}+M_{2}^{T}
\end{array}\right], \\
& \Psi_{2}=\left[\begin{array}{cc}
\bar{S}-U B F & 0 \\
-B F & 0
\end{array}\right], \\
& \Psi_{3}=\left[\begin{array}{cc}
-2 \bar{S} & \bar{S} \\
* & -\bar{R}-\bar{S}
\end{array}\right] .
\end{aligned}
$$

The state-feedback gain is then given by

$$
K=F M_{2}^{-T} \text {. }
$$

Remark 5. A tuning parameter matrix $U$ is employed in Theorems 4 and Corollary 2. By applying a numerical optimization algorithm [9], such as fminunc in the Optimization Toolbox, the tuning parameter matrix can be obtained.

\section{NUMERICAL EXAMPLES}

In the following, we will present three examples. Example 1 is given to compare with the result presented in [5], Example 2 is delivered to compare with the result presented in [12], and Example 3 is presented to illustrate the effectiveness of the designed state feedback controllers for NCSs with multiple-packet transmission. 
Example 1. Consider a system [5] with single-packet transmission

$$
\dot{x}(t)=\left[\begin{array}{cc}
0 & 1 \\
0 & -0.1
\end{array}\right] x(t)+\left[\begin{array}{c}
0 \\
0.1
\end{array}\right] u(t),
$$

and the controller is implemented through a network as $u(t)=K x(t-d(t))$, where $K=\left[\begin{array}{ll}-3.75 & -11.5\end{array}\right]$ and $0 \leq d(t) \leq h_{1}$. We can find the maximum allowable value of $h_{1}$ is 1.0081 by Corollary 1 , while the corresponding value of $h_{1}$ was 0.8695 by Lemma 1 (Corollary 1 in [5]).

Example 2. Consider the state-space plant transmitted by two packets in state channel, and by one packet in control channel. This example is borrowed from [12].

$$
\left[\begin{array}{c}
\dot{x}_{1} \\
\dot{x}_{2}
\end{array}\right]=\left[\begin{array}{cc}
-0.8 & 0.1 \\
0.2 & 0.05
\end{array}\right]\left[\begin{array}{l}
x_{1} \\
x_{2}
\end{array}\right]+\left[\begin{array}{l}
0 \\
1
\end{array}\right] u \text {. }
$$

For simplicity, we assume that $h_{1}=h_{2}$. The admissible upper bounds of $h_{i}$ were found to be $h_{i}=0.3102(i=1,2)$ and the corresponding feedback gain $K=\left[\begin{array}{ll}0.0756 & -4.1729\end{array}\right]$ in [12], while we can obtain the maximum upper bound $h_{i}=13.6784(i=1,2)$ and the corresponding feedback gain $K=\left[\begin{array}{ll}-0.0291 & 0.0762\end{array}\right]$ by Theorem 4 with $U=0.0001 I$. Obviously, it can be found that the method presented in this paper may provide less conservatism than the one presented in [12].

Example 3. Consider the following system

$$
\begin{aligned}
{\left[\begin{array}{c}
\dot{x}_{1} \\
\dot{x}_{2} \\
\dot{x}_{3} \\
\dot{x}_{4}
\end{array}\right]=} & {\left[\begin{array}{cccc}
-0.8 & 0.1 & 0.13 & 0.04 \\
0.2 & 0.05 & 0 & 0.12 \\
0 & -0.03 & -0.25 & 0.1 \\
0.1 & 0 & 0.02 & 0.03
\end{array}\right]\left[\begin{array}{l}
x_{1} \\
x_{2} \\
x_{3} \\
x_{4}
\end{array}\right] } \\
& +\left[\begin{array}{cc}
0 & 0.2 \\
1 & 0 \\
0.1 & 0 \\
0 & 1
\end{array}\right]\left[\begin{array}{l}
u_{1} \\
u_{2}
\end{array}\right]
\end{aligned}
$$

Case 1: Single-packet transmission in both state channel and control channel. Using Theorem 4 with $U=0.0001 I$, the maximum allowable transfer intervals is found as $h_{1}=$ 10.7434 , and the corresponding state-feedback gain is

$$
K=\left[\begin{array}{cccc}
-0.0122 & -0.0829 & 0.0181 & -0.0516 \\
-0.0128 & -0.0024 & -0.0181 & -0.0715
\end{array}\right] .
$$

Case 2: Single-packet transmission in control channel, and two-packet in state channel. It is assumed that $h_{1}=h_{2}$, $X_{1}=\left[\begin{array}{l}x_{1} \\ x_{2}\end{array}\right]$, and $X_{2}=\left[\begin{array}{l}x_{3} \\ x_{4}\end{array}\right]$. Then using Theorem 4 with $U=0.0001 I$, the maximum allowable transfer intervals are found as $h_{i}=9.0160(i=1,2)$, and the corresponding statefeedback gain is

$$
K=\left[\begin{array}{cccc}
-0.0536 & -0.0998 & -0.0324 & -0.0581 \\
0.0052 & 0.0096 & -0.0453 & -0.0818
\end{array}\right] .
$$

Case 3 : Two-packet transmission in both state and control channel. It is assumed that $h_{1}=h_{2}=h_{3}=h_{4}, X_{1}=\left[\begin{array}{l}x_{1} \\ x_{2}\end{array}\right]$,
$X_{2}=\left[\begin{array}{l}x_{3} \\ x_{4}\end{array}\right]$ and $U_{1}^{C}=u_{1}^{C}, U_{2}^{C}=u_{2}^{C}$. Using Theorem 4 with $U=0.0001 I$, it is found that, the maximum allowable transfer intervals are $h_{i}=8.9317(i=1,2,3,4)$, and the corresponding state-feedback gain is

$$
K=\left[\begin{array}{cccc}
-0.0519 & -0.0878 & -0.0917 & -0.0801 \\
0 & 0 & -0.0076 & -0.0627
\end{array}\right] .
$$

\section{CONCLUSION}

In this paper, a model for NCSs with multiple-packet transmission and packet dropout in both the sensor-to-controller channel and controller-to-actuator channel is given. A stability criterion and a new method for state feedback controller design are also presented by using LMI-based method. For the case of single-packet transmission, it has been shown that the newly proposed methods are less conservative than some existing results. The numerical examples have illustrated the effectiveness of the proposed methods.

\section{REFERENCES}

[1] G. M. Xie, and L. Wang, Stabilization of networked control systems with time-varying network-induced delay, Proceedings of the 43rd IEEE Conference on Decision and Control, 2004, pp. 3551-3556.

[2] Y. J. Pan, H. J. Marquez, and T. W. Chen, Remote stabilization of networked control systems with unknown time varying delays by LMI techniques, Proceedings of the 44th IEEE Conference on Decision and Control, 2005, pp. 1589-1594.

[3] S. S. Hu, and Q. X. Zhu, Stochastic optimal control and analysis of stability of networked control systems with long delay, Automatica, vol. 39, pp. 1877-1884, 2003.

[4] M. Yu, L. Wang, T. G. Chu, and F. Hao, An LMI approach to networked control systems with data packet dropout and transmission delays, Proceedings of the 43rd IEEE Conference on Decision and Control, 2004, pp. 3545-3550.

[5] D. Yue, Q. L. Han, and P. Chen, State feedback controller design of networked control systems, IEEE Transactions on Circuits and Systems-II:Express Briefs, vol. 51, no. 11, pp. 640-644, 2004.

[6] W. J. Kim, K. Ji, and A. Ambike, Networked real-time control strategies dealing with stochastic time delays and packet losses, Proceedings of the American Control Conference, 2005, pp. 621-626.

[7] J. L. Xiong and J. Lam, Stabilization of linear systems over networks with bounded packet loss, Automatica, vol. 43, pp. 80-87, 2007.

[8] P. Naghshtabrizi and J. P. Hespanha, Designing observer-type controllers for network control systems, Proceedings of the 44th IEEE Conference on Decision and Control, and the European Control Conference, 2005, pp. 848-853.

[9] X. M. Zhang, M. Wu, J. H. She, and Y. He, Delay-dependent stabilization of linear systems with time-varying state and input delays, Automatica, vol. 41, pp. 1405-1412, 2005.

[10] D. Yue, Q. L. Han, and J. Lam, Network-based robust $H_{\infty}$ control of systems with uncertainty, Automatica, vol. 41, pp. 999-1007, 2005.

[11] F. L. Lian, J. Moyne, and D. Tilbury, Analysis and modeling of networked control systems: MIMO case with multiple time delays, Proceedings of the American Control Conference, 2001, pp. 43064312.

[12] M. Yu, L. Wang, T. G. Chu, and F. Hao, Stabilization of networked control systems with data packet dropout and transmission delays: continuous-time case, European Journal of Control, vol. 11, pp. 4049, 2005.

[13] S. Boyd, L. El. Ghaoui, E. Feron, and V. Balakrishnan, Linear Matrix Inequality in Systems and Control Theory. Philadelphia, PA: SIAM, 1994.

[14] K. Gu, V. L. Kharitonov, and J. Chen, Stability of time-delay systems, Birkhauser, 2003. 